\title{
ENFOQUES Y ESTILOS DE APRENDIZAJE EN EDUCACIÓN SUPERIOR
}

\author{
LEARNING APPROACHES AND STYLES IN HIGHER EDUCATION
}

\author{
Fuensanta Hernández Pina* y Rosa María Hervás Avilés** \\ Universidad de Murcia
}

\section{RESUMEN}

Este artículo trata sobre los enfoques y estilos de aprendizaje de estudiantes universitarios. En el estudio se analizan sus perfiles de aprendizaje y como responden a las demandas educativas del entorno universitario. Igualmente se ha analizado la relación existentes entre los enfoques y los estilos de aprendizaje según las propuestas de Biggs y de Myers-Brigs. La muestra estudiada ha sido de 360 estudiantes de distintas titulaciones universitarias. Los resultados apunta a una relación entre enfoques y estilos de aprendizaje y una asociación de características de los estilos con los enfoques profundo y superficial.

Palabras clave: Enfoques y estilos de aprendizaje, motivos, estrategias, perfiles de aprendizaje en educación superior.

\begin{abstract}
In this article a study on learning approaches and styles in University students is presented. We analyse students profiles and how they respond to the academic demands of the university environment. We also analyse the relationship between the approaches to learning and learning styles proposed by Biggs and Myers-Briggs. The sample is made of 360 students of different university degrees. The results suggest there is a relationship between approaches to learning and learning styles as well as an association of features of the learning style with the deep and superficial approaches.
\end{abstract}

* Fuensanta Hernández Pina es Doctora en Pedagogía. Catedrática del Departamento MIDE de la Universidad de Murcia. Sus líneas de investigación se vinculan a la Evaluación Institucional; Evaluación de Centros Educativos. Enseñanza y aprendizaje universitarios; concepciones de la enseñanza y del aprendizaje y métodos de investigación en educación.

** Rosa María Hervás Avilés es Doctora en Pedagogía. Profesora en el Departamento MIDE de la Universidad de Murcia. Sus trabajos están relacionados con el estudio de los estilos de enseñanza-aprendizaje para la orientación psicopedagógica; aprendizaje estratégico y currículum escolar; enseñanza y aprendizaje universitarios, identificación y evaluación de alumnado de altas habilidades; mejora de los procesos de pensamiento en el aula. 
Key Words: Learning approaches and styles, motives, strategies, profiles of learning in higher education.

\section{Introducción}

Un cambio importante en la perspectiva de las investigaciones que en la últimas décadas se están llevando a cabo sobre el aprendizaje universitario, se enmarca dentro de una nueva perspectiva donde el contexto es el referente más importante. Al mismo tiempo, el nuevo modelo de educación superior se centra en el estudiante y en nuevos roles profesionales para el profesorado universitario en los que es importante la función tutorial y el conocimiento de las diferentes formas de aprender del alumnado, de sus enfoques y estilos de aprendizaje. Desde los años setenta las investigaciones en este campo intentan establecer un consenso sobre la forma en que los estudiantes universitarios comprenden y conocen su aprendizaje y estudio. Nos estamos refiriendo a la definición de los diferentes enfoques de aprendizaje que adopta el alumnado ante las tareas de aprendizaje. Esta área de estudio es conocida como Student Approaches to Learning (SAL).

Desde un punto de vista histórico, los primeros investigadores en realizar trabajos sobre aprendizaje universitario fueron Marton y Säljö (1976a, 1976b) en Suecia quienes acuñaron por primera vez los conceptos de enfoque profundo y enfoque superficial del aprendizaje para referirse a la forma en que los estudiantes universitarios se aproximaban a la lectura de artículos de investigación. A partir de estos estudios, otros investigadores con metodologías diferentes han obtenido resultados e implicaciones educativas similares. Nos referimos a los trabajos llevados a cabo por Biggs y su equipo de investigación en Australia y Hong Kong, y a Entwistle y Ramsden en Edimburgo.

Otro de los tópicos investigados en relación a las diversas formas de aprender y enseñar es el de los estilos educativos como preferencias individuales que influyen en la capacidad para aprender y que no están relacionados con las aptitudes sino con la forma en la que cada persona gusta de utilizar sus habilidades. En la actualidad existen diferentes modelos de estilos, fruto de las investigaciones surgidas desde comienzos del siglo XX en distintos ámbitos para conocer las causas por las que una persona difiere en su rendimiento, más allá de sus inteligencias. La variedad de modelos ha propiciado que en los últimos años proliferen estudios sobre las relaciones de unos modelos y otros como lo evidencian diferentes investigaciones, entre las que se encuentran las de Murray-Harvey (1994) quien analiza las relaciones entre el estilo de Dunn y Dunn y los enfoques de aprendizaje de Biggs. Asimismo, Sadler-Smith (1997) se refiere a los estilos de aprendizaje de Honey-Murdof, los enfoques de Entwistle y los estilos de apendizaje de Kolb. Igualmente, Snyder (2000) estudia las similitudes entre los estilos de aprendizaje, las inteligencias múltiples y el logro académico. Cano García (2000) correlaciona los estilos de aprendizaje de Kolb con los estilos intelectuales de Sternberg y la influencia de estos sobre el rendimiento académico. Zhang y Sternberg, (2000), Zhang $(2001,2004)$ se refieren a los enfoques de enseñanza y de aprendizaje de Biggs y los estilos intelectuales. Igualmente Zhang, (2000) compara los estilos de personalidad de Holland y el modelo de Costa y McCrae (2003) con los estilos intelectuales de Sternberg y con los enfoques de aprendizaje de Biggs. Recientemente Dunn, Dening y Lovelace (2001) analizan cómo utilizar los estilos de aprendizaje para mejorar el rendimiento de los estudiantes y comparan el modelo de estilo de aprendizaje de Dunn, Dunn y Price 
con el de Inteligencias Múltiples de Gardner y finalmente Hervás y Castejón (2003) comparan los estilos epistémicos de Royce, Powell y Rancourt y los Tipos psicológicos de Jung.

En esta línea, este trabajo presenta algunos de los resultados obtenidos en una investigación sobre la relación entre los enfoques y los estilos de aprendizaje de estudiantes universitarios de distintas titulaciones. Pretendemos conocer la existencia o no de un perfil predominante en una muestra de estudiantes universitarios, en función de los diferentes estilos y enfoques de aprendizaje. Igualmente, establecemos la relación entre los enfoques de aprendizaje fundamentados en las investigaciones de Biggs en Australia y los estilos de aprendizaje basados en el modelo teórico de Myers-Brigs .

\section{Enfoques de aprendizaje}

Término acuñado para referir los procesos que surgen de las percepciones que los estudiantes tiene de las tareas académicas influenciadas por sus rasgos de personalidad. La investigación sobre el aprendizaje, desde la propia perspectiva del alumnado, iniciada en Suecia por F. Marton y posteriormente desarrollada por N. Entwistle en Lancaster y J. Biggs en Australia, entre otros, ha aportado una nueva dimensión al estudio del aprendizaje en su verdadero contexto creando un corpus de conocimientos sólido que permite la reflexión y transformación de muchos principios teóricos del aprendizaje en propuestas para la práctica educativa. Parece existir anuencia en señalar que los enfoques de aprendizaje están formados por dos componentes: la motivación o intenciones del estudiante para aprender, y las estrategias que utiliza en su proceso de aprendizaje.La forma de aproximarse al aprendizaje va a depender de los motivos y de las intenciones, de las características personales, de los conocimientos que se poseen y del uso que se haga de los conocimientos previos; todo ello dentro de un escenario educativo determinado. De esta relación o congruencia entre intención/motivo y proceso/estrategia dependerá en gran medida el resultado del aprendizaje. Y es precisamente esta relación intención-proceso-resultado la que subyace al propio concepto de enfoque de aprendizaje (Biggs, 1987, Hernández Pina, 1993, 1999, 2002).

Como evidencian distintos estudios, los enfoques de aprendizaje pueden ser descritos y analizados a dos niveles de generalidad:

a) un enfoque puede describir la combinación entre la intención y el proceso utilizado por los estudiantes para abordar una tarea particular en un momento determinado, y

b) un enfoque puede también referirse a la forma en que un estudiante de forma consistente, se enfrenta a la mayoría de las tareas o materias de aprendizaje.

La motivación y las metas determinan la manera de afrontar los procesos implicados en el aprendizaje. Se trata de un proceso complejo de metaaprendizaje (Biggs, 1987) que implica:

a) que el alumnado esté seguro de sus propios recursos cognitivos en relación a las demandas de las tareas de aprendizaje y

b) que controle la selección y utilización de determinadas estrategias.

El aprendizaje se convierte, por tanto, en una interacción con el entorno y en un cambio conceptual, y no, como se ha venido manteniendo durante mucho tiempo, en una mera adquisición de información (perspectiva ecológica). 
Algunos autores identifican y describen tres dimensiones que caracterizan la forma habitual en que el alumnado universitario se enfrenta o aproxima a sus tareas de aprendizaje o estudio. Son constructos que se han configurado a lo largo de los últimos veinte años: enfoque profundo, enfoque superficial y enfoque estratégico o de alto rendimiento. Es lo que Richardson (1994), describe como tres formas básicas de aproximación al aprendizaje por parte de los estudiantes: a) una orientación hacia la comprensión del significado de los materiales de estudio, b) otra orientada hacia la reproducción de los materiales de estudio con fines académicos y evaluativos, y c) una orientación hacia la obtención del éxito académico basado en altas calificaciones. Sin embargo, investigaciones posteriores como las de Kember (1996, 2000) y Hernández Pina (1999) parecen poner de manifiesto que los enfoques de aprendizaje forman parte de un continuo en el que los polos opuestos se situarían en el enfoque profundo y superficial mientras que en las fases intermedias se acomodan una variedad de enfoques en función del peso que tenga la intención de comprender o memorizar para el estudiante y las demandas del escenario educativo (Hernández Pina y otros, 2002: 488). Para Kember la forma en que el estudiante combina la memorización y la comprensión dan lugar a dicho continuo. El estudiante se situaría dentro de dicho continuo en el lugar caracterizado por los motivos o intenciones y por las estrategias empleadas. Los perfiles de los enfoques de aprendizaje, descritos en anteriores estudios (Hernández Pina, 1993, 1999), no son características psicológicas estables, sino que el enfoque adoptado por el estudiante para una tarea particular estará en función de la naturaleza de dicha tarea, de la evaluación, del método de enseñanza, de la percepción que el estudiante desarrolle, de la relevancia del curso, del interés del estudiante, de su estilo de aprendizaje (Laurillard, 1984, Ramsden, 1984, Gibbs, 1992, Biggs, 1999, Hernández Pina, 1993, Kember, 2000). Igualmente el proceso instruccional influyen en el enfoque de aprendizaje de los estudiantes. En consecuencia, las actitudes y las creencias del profesor son relevantes para el aprendizaje de los estudiantes (Kember y Gow, 1993).

Además, la categorización de los enfoques de aprendizaje permite conocer y describir el modo en que los estudiantes aprenden sea cual sea el lugar en que lo hagan. Ha permitido, igualmente, establecer unos modelos de enseñanza-aprendizaje comprensivos que ayudan a establecer qué factores determinan la calidad del aprendizaje (Biggs, 1987 y Entwistle, 1998), y al mismo tiempo han posibilitado la apertura de un camino de comunicación entre investigadores procedentes de distintos países. En este sentido, Biggs (1999) elabora el modelo 3P de enseñanza-aprendizaje que incluye tres factores relacionados con la calidad del aprendizaje de forma directa, indirecta e intencionada. En el modelo aparece en primer lugar los factores de Presagio en los que se que consideran los factores del estudiante y los factores del contexto de la enseñanza y la institución, incluyendo las concepciones de la enseñanza y del aprendizaje practicadas por los profesores (Trigwell y Prosser, 1996). Los factores de Proceso vienen representados por los enfoques de aprendizaje que se derivan de los factores de presagio. Por último el factor Producto, que se refiere a la calidad del aprendizaje y la implicación del estudiante en dicho aprendizaje (Hernández Pina, 1993, 1999).

Para evaluar los enfoques de aprendizaje se han elaborando diversos cuestionarios como por ejemplo Approaches to Study Inventroy, (ASI) y Biggs (1987) y Study Process Questionnarie (SPQ) que tratan de operativizar dichos enfoques. Las respuestas a tales cuestionarios informan, del mismo modo, acerca de la calidad del ambiente de enseñanza debido a que el enfoque adoptado por un estudiante va a estar en función de las demandas y las tareas requeridas en dicho ambiente de enseñanza. Esto significa que estos cuestionarios se han 
convertido en instrumentos válidos para evaluar dichos ambientes (Kember et al 1998, o Biggs, 1999). El cuestionario de procesos de aprendizaje ha sido adaptado y aplicado a dos muestras españolas con resultados muy similares a los obtenidos por otros estudios, tanto en su fiabilidad como en su validez de constructo, (Hernández Pina, 1999).

\section{Los tipos psicológicos como estilos de aprendizaje}

Entendemos los estilos de aprendizaje como las preferencias, los procesos o formas particulares que tenemos para aprender (Hervás, 2003). Con este sentido, y basándose en la teoría de Tipos de Jung, distintos autores (Myers y Briggs, 1943, 1976, Myers, 1992, Myers y McCaulley, 1985), desarrollan el MBTI (Myer-Briggs Type Indicator) y amplían sus aplicaciones. Estas autoras, proponen 16 de tipos psicológicos resultantes de las posibles combinaciones de unas preferencias asociadas a:

1) Dos funciones para percibir y adquirir información:

a) la sensorial (S) relacionada con quienes perciben lo evidente, de una manera secuencial, centrándose en la realidad, en los hechos, en lo concreto y en su aplicación práctica, orientados al presente,

b) la intuitiva $(\mathrm{N})$ referida a quienes perciben de forma abstracta y global; valorando los presentimientos; interesándose por el futuro y lo posible utilizando el ingenio y la creatividad.

2) Dos funciones para tomar decisiones:

c) la reflexión (T) asociada a quienes tienden a decidir de forma objetiva e impersonal basándose en la lógica; suelen ser escépticos y firmes en sus decisiones;

d) el sentimiento (F) función que implica decisiones personales, subjetivas, fundamentadas en un sistema de valores propio; igualmente implica empatía y preocupación por complacer a los otros.

3) Dos actitudes o formas de relacionarse con el exterior:

e) la extraversión (E) orientada a la interacción con personas y cosas; relacionada con la acción; quienes adoptan una actitud extravertida utilizan el entorno para estimularse, tienen facilidad para comunicarse, son sociables, les gusta trabajar con los demás y necesitan verbalizar sus pensamientos; y

f) la introversión (I) implica concentrar la atención en el mundo interior; supone la reflexión, la concentración en las propias ideas, emociones e impresiones.

4) Dos actitudes que determinan las funciones que predominan en la actividad de la persona con su mundo exterior:

g) la actitud perceptiva (P) supone que en el estilo de aprendizaje predomina cualquiera de las funciones perceptivas $(\mathrm{S}-\mathrm{N})$. Incluye flexibilidad, dedicar tiempo a obtener información, a observar. Conlleva cierta improvisación dejando que las cosas ocurran, que sigan su camino;

h) la actitud juicio $(\mathrm{J})$ incluye el predominio de las funciones utilizadas para tomar decisiones (reflexiva o emotivamente). Implica planificación, estructuración, previsión, autorregulación, organización, establecimiento de metas y objetivos, con- 
trol del aprendizaje. El juicio supone obtener conclusiones de lo percibido. Significa tomar decisiones, evaluar, elegir y seleccionar una respuesta después de percibir un estímulo. Las personas usan su función dominante fundamentalmente cuando se encuentran en un ambiente cómodo para ellas (extraversión-introversión). La combinación resultante de funciones y actitudes da como resultado 16 estilos de aprendizaje tal y como muestra la figura 1.

\begin{tabular}{|l|l|l|l|l|l|}
\hline \multicolumn{7}{|c|}{ DIMENSIONES Y TIPOS PSICOLÓGICOS SEGÚN LA } \\
\hline Estraversión (E) & & & & & Intervención (I) \\
\hline Sensación (S) & & & & & Intuición (N) \\
\hline Pensamiento (T) & & & & & Sentimiento (F) \\
\hline Juicio (J) & & & & & Percepción (P) \\
\hline & ISTJ & ISFJ & INFJ & INTJ & \\
\hline & ISTP & ISFP & INFP & INTP & \\
\hline & ESTP & ESFP & ENFP & ENTP & \\
\hline & ESTJ & ESFJ & ENFJ & ENTJ & \\
\hline
\end{tabular}

FIGURA 1.

Modelo de Myers-Briggs. Dimensiones y tipos psicológicos.

La teoría del tipo mantiene que las personas nacen con una predisposición hacia unas funciones en lugar de otras. Los estudiantes están más interesados en el dominio de su función preferida, se sienten motivados al utilizarla y perfeccionarla, se sienten competentes cuando pueden ejercerla bien. La utilización de estas funciones se amplía a distintas actividades incorporando conductas, rasgos y habilidades asociadas al desarrollo de la misma. En este modelo, el entorno es extremadamente importante porque los factores ambientales pueden fomentar el incremento de las preferencias naturales de cada persona, o puede desalentar su inclinación natural mediante actividades menos satisfactorias y menos motivadoras, dificultando el desarrollo de las habilidades. La ampliación del tipo de estilo es un proceso que dura toda la vida. Mediante él se adquiere un mayor dominio de las funciones de percepción y del juicio.

A partir de todas estas consideraciones, en este trabajo presentamos las relaciones encontradas entre los enfoques de aprendizaje fundamentados en las investigaciones de Biggs y los estilos de aprendizaje basados en el modelo teórico de Myers-Brigs.

\section{Metodología}

\section{Objetivos de la investigación}

Esta investigación tiene como objetivo general identificar y relacionar los enfoques y los estilos de aprendizaje en estudiantes universitarios. Los objetivos específicos que se desprenden son: 
1) analizar los enfoques de aprendizaje de los estudiantes universitarios;

2) estudiar descriptivamente los estilos de aprendizaje de los estudiantes universitrios;

3) establecer las relaciones de los enfoques y los tipos psicológicos como estilos de aprendizaje;

4) estudiar mediante una prueba de independencia la relación entre las variables del CPE y las variables del MBTI;

5) comparar mediante una prueba no paramétrica para dos muestras independientes los promedios de los enfoques y de los estilos de aprendizaje.

\section{Población y muestra}

La población de referencia en nuestra investigación ha sido el colectivo de estudiantes universitarios de distintas titulaciones matriculados durante el curso académico 2000-2001. El número de estudiantes que han participado en este estudio ha sido de 360 procedentes de las titulaciones de Pedagogía $(n=85)$; Psicopedagogía $(n=, 129)$; Historia $(n=99)$ e Informática $(n=47)$. En cuanto al género el $27 \%$ es masculino y el $63 \%$ femenino. El método de muestreo que hemos utilizado ha sido no aleatorio y con un carácter incidental. Es decir, los cuestionarios fueron cumplimentados voluntariamente por todos los estudiantes que asistieron a clase el día de la aplicación de las pruebas.

\section{Instrumentos}

Se han utilizado dos instrumentos:

a) Study Process Questionnaire (SPQ) (Cuestionario sobre procesos en el estudio (CPE) (Biggs, 1987) para evaluar los enfoques de aprendizaje. Este cuestionario ha sido adaptado y aplicado a tres muestras españolas con resultados muy similares a los obtenidos por otros estudios, tanto en su fiabilidad como en la validez de constructo (Hernández Pina, 1993, 1999, 2003). El cuestionario consta de 42 preguntas, cada una de ellas acompañada de una escala tipo Likert con 5 niveles. Mide los tres enfoques (superficial, profundo y de alto rendimiento). Cada enfoque está compuesto por 14 preguntas, 7 referidas a motivo y 7 referidas a estrategias.

b) Igualmente se ha utilizado el indicador de tipo de Myers-Briggs (MBTI. Forma G) (Myers y Briggs, 1943,1976) que consta de 126 preguntas acerca de las preferencias de los sujetos sobre su forma de percibir o de actuar ante diferentes situaciones. El formato general de las preguntas obliga a elegir una entre dos alternativas. A partir de las respuestas de los sujetos se obtienen unas puntuaciones de preferencia en cada una de las 8 subescalas: extraversión (E), introversión (I), sensación (S), intuición $(\mathrm{N})$, reflexión $(\mathrm{T})$, sentimiento $(\mathrm{F})$, juicio $(\mathrm{J})$ y percepción $(\mathrm{P})$. También es posible calcular una puntuación de preferencia entre cada dos escalas contrapuestas extraversión-introversión, sensación-intuición, reflexión-emoción y juicio-percepción. La combinación de estas funciones y actitudes da lugar a 16 tipos que difieren en el desarrollo de cada preferencia y en la actitudes de Introversión (I) y Extraversión (E), Percepción y Juicio que se adoptan con respecto a la realidad. Se trata de un instru- 
mento de evaluación ampliamente adaptado a nuestra población y con numerosas investigaciones que acreditan su utilidad y validez.

\section{Análisis y resultados}

El análisis se ha realizado utilizando el paquete estadístico SPSS versión 12.01.

\section{Identificación de los enfoques de aprendizaje de los estudiantes}

\section{Objetivo primero: analizar los enfoques de aprendizaje de los estudiantes universitarios}

Una vez que los participantes cumplimentaron el cuestionario CPE, se calculó su puntuación en cada uno de los tres enfoques. Para establecer su perfil, cada estudiante fue identificado en el enfoque predominante en el que obtuvo mayor puntuación. A continuación, se calculó el porcentaje de estudiantes que utilizaba predominantemente cada enfoque. En la tabla 1 observamos que el 47,50 de los estudiantes tienen un enfoque profundo; el $42,22 \%$ un enfoque superficial, mientras que el $10,28 \%$ manifiestan un enfoque de alto rendimiento.

TABLA 1: Distribución porcentual de estudiantes por enfoque de aprendizaje.

\begin{tabular}{|c|c|c|}
\hline SA & DA & AA \\
\hline $42,22 \%$ & $7,50 \%$ & $10,28 \%$ \\
\hline
\end{tabular}

En cuanto al análisis por titulaciones, observamos en la tabla 2 como el porcentaje más alto de los estudiantes de Pedagogía se sitúa en el enfoque superficial con un 57,65\% seguido del enfoque profundo 32,94 y de alto rendimiento, con 9,41\%. Estos datos son similares a los de la titulación de Informática en los que también el porcentaje más alto de enfoque predominante lo encontramos en enfoque superficial con un 59,57\% seguido de enfoque profundo con un $23,40 \%$ y enfoque de alto rendimiento con un $17,02 \%$. El porcentaje más elevado de estudiantes de Psicopedagogía se relaciona con el enfoque profundo (DA) con un $51,16 \%$ seguido del superficial (SA) con un $38,76 \%$ y un $10,08 \%$ para el de alto rendimiento (AA). Finalmente la titulación de Historia el porcentaje más alto es el del enfoque

TABLA 2: Distribución porcentual de enfoques de aprendizaje por titulaciones.

\begin{tabular}{|l|c|c|c|}
\hline & SA & DA & AA \\
\hline PEDAGOGÍA & $57,65 \%$ & $32,94 \%$ & $9,41 \%$ \\
\hline PSICOPEDAGOGÍA & $38,76 \%$ & $51,16 \%$ & $10,08 \%$ \\
\hline HISTORIA & $25,25 \%$ & $66,67 \%$ & $8,08 \%$ \\
\hline INFORMÁTICA & $59,57 \%$ & $23,40 \%$ & $17,02 \%$ \\
\hline
\end{tabular}


profundo (DA) que se eleva a $66,67 \%$ seguido de un $25,25 \%$ en el enfoque superficial (SA) y un $8,08 \%$ en el de alto rendimiento (AA).

Como vemos las dos titulaciones de Pedagogía e Informática tienen un porcentaje elevado de estudiantes con un enfoque superficial (SA), frente a Psicopedagogía e Historia donde los porcentajes más altos se sitúan en enfoque profundo (DA).

\section{Identificación de los estilos de aprendizaje}

Objetivo segundo: estudiar descriptivamente los estilos de aprendizaje de los estudiantes universitarios

Por lo que respecta a los resultados obtenidos en relación a los tipos psicológicos como estilos de aprendizaje (tabla 3) éstos indican claras preferencias de los estudiantes hacia la extraversión $(62,78 \%)$, la percepción sensorial $(71,94 \%)$, la toma de decisiones reflexiva $(67,78 \%)$ y una actitud perceptiva $(50,83 \%)$.

TABLA 3: Distribución global porcentual de las dimensiones de los estilos de aprendizaje.

\begin{tabular}{|c|c|c|c|c|c|c|c|}
\hline $\mathbf{E}$ & $\mathbf{I}$ & $\mathbf{S}$ & $\mathbf{N}$ & $\mathbf{T}$ & $\mathbf{F}$ & $\mathbf{J}$ & $\mathbf{P}$ \\
\hline $62,78 \%$ & $37,22 \%$ & $71,94 \%$ & $28,06 \%$ & $67,78 \%$ & $32,22 \%$ & $49,17 \%$ & $50,83 \%$ \\
\hline
\end{tabular}

Los resultados por titulaciones (tabla 4) indican la existencia de:

- Claras preferencias hacia la extraversión en todas las titulaciones, sobre todo en Psicopedagogía, excepto en Informática cuyos estudiantes manifiestan una ligera mayoría hacia la introversión.

- En todas las titulaciones los estudiantes manifiestan preferir la percepción sensorial, sobre todo en Pedagogía.

- Los estudiantes manifiestan que prefieren tomar decisiones de una manera objetiva y reflexiva en todas las titulaciones, sobre todo en Historia e Informática.

- Finalmente, en cuanto a las actitudes que marcan la dinámica del tipo solamente en Pedagogía predominan las funciones relacionadas con la toma de decisiones $(\mathrm{J})$ mientras que en el resto de las titulaciones los estudiantes manifiestan una preferencia por la funciones perceptivas.

TABLA 4: Distribución porcentual de las dimensiones de los Tipos Psicológicos en Pedagogía, Psicopedagogía, Historia e Informática.

\begin{tabular}{|l|c|c|c|c|c|c|c|c|}
\hline \multicolumn{1}{|c|}{ TITULACIONES } & E & I & S & N & T & F & J & P \\
\hline PEDAGOGÍA & $63,53 \%$ & $36,47 \%$ & $81,18 \%$ & $18,82 \%$ & $55,29 \%$ & $44,71 \%$ & $58,82 \%$ & $41,82 \%$ \\
\hline PSICOPEDAGOGÍA & $72,09 \%$ & $27,91 \%$ & $69,77 \%$ & $30,23 \%$ & $62,02 \%$ & $37,98 \%$ & $48,06 \%$ & $51,94 \%$ \\
\hline HISTORIA & $56,57 \%$ & $43,43 \%$ & $68,69 \%$ & $31,31 \%$ & $81,82 \%$ & $18,18 \%$ & $44,44 \%$ & $55,56 \%$ \\
\hline INFORMÁTICA & $48,94 \%$ & $51,06 \%$ & $68,09 \%$ & $31,91 \%$ & $76,60 \%$ & $23,40 \%$ & $44,68 \%$ & $55,32 \%$ \\
\hline
\end{tabular}




\section{Correspondencia entre los enfoques y los estilos de aprendizaje}

\section{Objetivo tercero: relacionar los enfoques y los estilos de aprendizaje}

Por lo que respecta al tercer objetivo de nuestra investigación y a la pregunta de si están relacionadas las variables de los estilos de aprendizaje y los enfoques de aprendizaje hemos realizado, en primer lugar, un análisis descriptivo y exploratorio cuyos resultados nos indica que los enfoques y los estilos de aprendizaje se corresponden de la siguiente manera:

- Los estudiantes con un enfoque superficial tienen mayoritariamente (78,95\%) una percepción sensorial, son extravertidos $(63,82 \%)$, toman decisiones reflexivamente $(58,55 \%)$ y utilizan preferentemente las funciones perceptivas $(57,24 \%)$ (tabla 5).

TABLA 5: Distribución porcentual de las dimensiones de los Tipos Psicológicos de los estudiantes con un enfoque superficial.

\begin{tabular}{|c|c|c|c|}
\hline \multicolumn{3}{|c|}{ SA } \\
\hline Percepción sensorial $(\mathrm{S})$ & Extraversión (E) & Decisiones Reflexión $(\mathrm{T})$ & Percepción (P) \\
\hline $78,95 \%$ & $63,82 \%$ & $58,55 \%$ & $57,25 \%$ \\
\hline
\end{tabular}

- El alumnado con un enfoque profundo es fundamentalmente reflexivo en su toma de decisiones $(75,55 \%)$, sensorial en su percepción $(62,57 \%)$, y extravertido en su relación con los demás. Igualmente estos estudiantes utilizan preferentemente las funciones de toma de decisiones (J) (tabla 6).

TABLA 6: Distribución porcentual de las dimensiones de los Tipos Psicológicos de los estudiantes con un enfoque profundo.

\begin{tabular}{|c|c|c|c|}
\hline \multicolumn{4}{|c|}{ DA } \\
\hline Decisiones Reflexión (T) & Percepción sensorial (S) & Extraversión (E) & Juicio (J) \\
\hline $75,55 \%$ & $62,57 \%$ & $75,44 \%$ & $50,29 \%$ \\
\hline
\end{tabular}

- Los estudiantes con un enfoque de alto rendimiento son mayoritariamente sensoriales $(86,49 \%)$, reflexivos $(70,27 \%)$, utilizan la gran mayoría $(70,27 \%)$ las funciones de juicio con mayor comodidad que las de percepción, y son poco más de la mitad de estos estudiantes son introvertidos (50,05\%) (tabla 7 ).

TABLA 7: Distribución porcentual de las dimensiones de los Tipos Psicológicos de los estudiantes con un enfoque de alto rendimiento.

\begin{tabular}{|c|c|c|c|}
\hline \multicolumn{3}{|c|}{ AA } \\
\hline Percepción sensorial (S) & Decisiones Reflexión (T) & Juicio (J) & Introversión (I) \\
\hline $86,49 \%$ & $70,27 \%$ & $70,27 \%$ & $50,05 \%$ \\
\hline
\end{tabular}


Podemos concluir:

- Los estudiantes con un enfoque superficial se inclinan por las funciones perceptivas como dominantes.

- Los estudiantes con un enfoque profundo y de alto rendimiento utilizan preferentemente las funciones relacionadas con la toma de decisiones.

- Los estudiantes con un enfoque superficial permanecen más tiempo percibiendo y observando, mientras que los de enfoque profundo y de alto rendimiento dedican menos tiempo a la fase de percepción para llegar más pronto a la toma de decisiones y a las conclusiones de un problema.

\section{Relación entre las variables del CPE (Cuestionario de procesos de estudio) y las variables del MBTI (Indicador de tipos de Myers-Briggs)}

\section{Objetivo cuarto: estudiar mediante una prueba de independencia la relación entre las variables del CPE y las variables del MBTI}

Para el análisis de este objetivo hemos aplicado una prueba de independencia o prueba chi cuadrado que nos permite relacionar algunas de las variables del CPE con las variables del MBTI.

Los resultados nos indican las siguientes relaciones:

1. Los estudiantes con un enfoque profundo se relacionan con las variables de los estilos de aprendizaje siguientes:

\section{Motivos}

- Quienes siempre tienen la necesidad de descubrir la verdad, perciben intuitivamente $(p<0,05)$.

- Aquellos estudiantes que siempre encuentran tan atractivo estudiar ciertas materias como leer una novela son introvertidos $(p<0,05)$.

\section{Estrategias}

- Los estudiantes que casi siempre utilizan la estrategia de relacionar la información nueva con lo que ya saben, perciben intuitivamente y toman decisiones reflexivamente, mientras que los que utilizan esta estrategia algunas veces perciben sensorialmente y toman decisiones emotivamente $(p<0,05)$.

- El alumnado que frecuentemente utilizan la estrategia de formarse una opinión personal de lo que estudian para quedar satisfechos, toman decisiones reflexivamente, mientras que los estudiantes que la utilizan algunas veces toman decisiones afectivamente. $(p<0,05)$.

- Quienes siempre intentan relacionar lo que han aprendido en una materia con lo que ya saben de otras, perciben intuitivamente $(p<0,01)$.

Como podemos apreciar las variables de los estilos de aprendizaje que más se relacionan tanto con los motivos como con las estrategias de quienes tiene un enfoque profundo son: la percepción intuitiva, la toma de decisiones reflexiva y la introversión. 
2. Los estudiantes con un enfoque superficial se relacionan con las variables de los estilos de aprendizaje siguientes:

\section{Motivos}

- El alumnado al que le desagrada estudiar una carrera pero lo hace porque el resultado final le merece la pena, siempre percibe sensorialmente y nunca intuitivamente $(p<0,05)$.

- Aquellos estudiantes que consideran que están en la universidad para conseguir un buen puesto de trabajo, siempre perciben sensorialmente y nunca intuitivamente $(p<0,05)$.

\section{Estrategias}

- Quienes siempre estudian los apuntes y lo que en clase se señala porque consideran que buscar información complementaria es una tontería son extraverti$\operatorname{dos}(p<0,05)$.

- Los estudiantes que emplean poco tiempo en estudiar aquello que no va a salir en los exámenes, siempre prefieren los procesos de percepción a los de toma de decisiones $(p<0,05)$.

Los estudiantes que frecuentemente prefieren aceptar las ideas del profesorado sin cuestionarlas perciben sensorialmente y nunca intuitivamente $(p<0,05)$.

En este caso los motivos y las estrategias del enfoque superficial se relacionan con las funciones perceptivas y concretamente con la percepción sensorial y secuencial frente a la intuitiva que es más abstracta y global.

3. Los estudiantes con un enfoque de logro o alto rendimiento se relacionan con las variables de los estilos de aprendizaje siguientes:

\section{Motivos}

- Los estudiantes que frecuentemente pretenden sacar las mejores calificaciones para conseguir los mejores puestos de trabajo, perciben sensorialmente y prefieren las funciones de tomar decisiones a las perceptivas $(p<0,05)$.

- Quienes frecuentemente desean destacar en todas las asignaturas perciben intuitivamente y nunca sensorialmente $(p<0,05)$.

- Quienes siempre estarían dispuestos a sacrificar la popularidad que tuvieran con los compañeros para tener éxito, siempre son introvertidos y nunca extravertidos $(p<0,05)$.

- Los estudiantes para los que lograr buenas notas es un juego competitivo en el que le gusta ganar siempre prefieren los procesos perceptivos y nunca los de tomar decisiones $(p<0,05)$.

\section{Estrategias}

- El alumnado que siempre estudian sistemáticamente y revisan los apuntes con regularidad prefieren los procesos de toma de decisiones $(p<0,05)$.

- Los estudiantes que frecuentemente hacen las tareas cuanto antes son extravertidos y prefieren los procesos de toma de decisiones $(p<0,05)$. 
- Quienes siempre tienen los apuntes estructurados y bien organizados prefieren los procesos de toma de decisiones, mientras que los que los tienen algunas veces prefieren las perceptivas $(p<0,05)$.

En este caso existe más variedad de respuesta en la relación entre las variables de estilos de aprendizaje y con los motivos y las estrategias de alto rendimiento.

Las estrategias de alto rendimiento relacionadas con las variables de estilos de aprendizaje lo hacen con las funciones de toma de decisiones del MBTI.

\section{Comparación de promedios de los enfoques de aprendizaje y estilos de aprendizaje}

\section{Objetivo quinto: comparar mediante una prueba no paramétrica para dos muestras independientes los promedios de los enfoques y de los estilos de aprendizaje}

Se han comparado los promedios de las variables de los enfoques superficial (SA), profundo (DA) y de alto rendimiento (AA) para las cuatro dimensiones de MBTI: Extraversión-Introversión; Sensación-Intuición; Reflexión-Emoción y, Juicio-Percepción. Para ello realizamos una prueba no parámetrica para dos muestras independientes, concretamente la prueba U de Mann-Whitney. Consideramos grupos independientes los elementos con MBTI igual a 1 y MBTI igual a 2. Como variables dependientes, para las que queremos ver si existen diferencias en promedio para los dos grupos anteriormente señalados: SA (enfoque superficial) DA (enfoque profundo) y AA (enfoque de alto rendimiento).

Los resultados que se obtienen, son los siguientes:

1. Para la Extraversión-Introversión no existen diferencias significativas en promedio para los dos grupos considerados $(p>0.05)$.

2. En cuanto a la Sensación-Intuición, existen diferencias estadísticamente significativas en promedio para los grupos mencionados, para SA $(p<0.05)^{*}$ y DA $(p<0.01)^{*}$. Así, tenemos que el promedio en el enfoque superficial para los estudiantes que perciben intuitivamente es más bajo que el promedio en el enfoque superficial para los estudiantes que perciben sensorialmente. Igualmente el promedio en el enfoque profundo para los estudiantes que perciben sensorialmente es menor que el promedio en el enfoque profundo de los estudiantes que perciben intuitivamente (tabla 8).

3. Si consideramos la toma de decisiones reflexiva o emotiva (Reflexión-Sentimiento) los resultados indican que existen diferencias estadísticamente significativas en promedio para los grupos mencionados. Concretamente en los enfoques superficial $(p<0,05)^{*}$ y profundo $(p<0,01)^{*}$. Así, el promedio en el enfoque superficial de los estudiantes que toman decisiones reflexivamente es menor que el promedio en el mismo enfoque de los estudiantes que toman decisiones afectivamente. De igual manera el promedio en el enfoque profundo de los estudiantes que toman decisiones reflexivamente es mayor que el promedio en este mismo enfoque de los estudiantes que toman decisiones afectivamente (tabla 9). 
TABLA 8: Medianas ( rango) y valores $Z$ para enfoques $y$ dimensiones (S) y (N) de los estilos de aprendizaje.

\begin{tabular}{|l|c|c|c|}
\cline { 2 - 4 } \multicolumn{1}{c|}{} & \multicolumn{3}{c|}{$\begin{array}{c}\text { Mediana (Rango) y valores } \mathbf{Z} \text { para enfoques y } \\
\text { dimensiones de los estilos de aprendizaje }\end{array}$} \\
\hline \multicolumn{1}{|c|}{ MBTI } & SA & DA & AA \\
\hline Sensación & $45(40)$ & $44(44)$ & $38(11)$ \\
\hline Intuición & $43(33)$ & $47(33)$ & $37(38)$ \\
\hline$Z$ & $-2,326^{*}$ & $-3,411 * *$ & -0.338 \\
\hline \multicolumn{4}{|c|}{$* 0.05, * * \mathrm{p}<0.01, * * * \mathrm{p}<0.01$} \\
\hline
\end{tabular}

TABLA 9: Medianas (rango) y valores $Z$ para enfoques $y$ dimensiones $(T)$ y $(F)$ de los estilos de aprendizaje.

\begin{tabular}{|l|c|c|c|}
\cline { 2 - 4 } \multicolumn{1}{c|}{} & \multicolumn{3}{c|}{$\begin{array}{c}\text { Medianas (Rango) y valores } Z \text { para enfoques y } \\
\text { dimensiones de los estilos de aprendizaje }\end{array}$} \\
\hline \multicolumn{1}{|c|}{ MBTI } & SA & DA & AA \\
\hline Reflexión & $44(39)$ & $46(40)$ & $39(38)$ \\
\hline Emoción & $47(32)$ & $44(37)$ & $36,5(43)$ \\
\hline$Z$ & $-2,949 *$ & $-2,502 * *$ & -1.810 \\
\hline \multicolumn{4}{|c|}{$* 0.05, * * \mathrm{p}<0.01, * * * \mathrm{p}<0.01$} \\
\hline
\end{tabular}

4. Teniendo en cuenta las actitudes de juicio y percepción, existen diferencias estadísticamente significativas en el promedio para los grupos mencionados, concretamente en el enfoque de alto rendimiento Así, el promedio en el enfoque de alto rendimiento (AA) de los estudiantes que se sienten más cómodos tomando decisiones que percibiendo es mayor que el promedio en el mismo enfoque de los estudiantes que prefieren percibir a tomar decisiones (tabla 10).

TABLA 10: Medianas ( rango) y valores $Z$ para enfoques $y$ dimensiones $(J)$ y $(\mathrm{P})$ de los estilos de aprendizaje.

\begin{tabular}{|l|c|c|c|}
\cline { 2 - 4 } \multicolumn{1}{c|}{} & \multicolumn{3}{c|}{$\begin{array}{c}\text { Medianas (Rango) y valores } Z \text { para enfoques y } \\
\text { dimensiones de los estilos de aprendizaje }\end{array}$} \\
\hline \multicolumn{1}{|c|}{ MBTI } & SA & DA & AA \\
\hline Juicio & $45(40)$ & $45(43)$ & $40(36)$ \\
\hline Percepción & $46(33)$ & $46(42)$ & $37(43)$ \\
\hline$Z$ & $-2,65$ &,- 406 & $-2.838^{*}$ \\
\hline \multicolumn{4}{|c|}{$* 0.05, * * \mathrm{p}<0.01, * * * \mathrm{p}<0.01$} \\
\hline
\end{tabular}




\section{Conclusiones}

a) Casi la totalidad de la muestra estudiada tiene un enfoque de aprendizaje profundo(47,50\%), y un enfoque superficial $(42,22 \%)$. Solamente el $10,28 \%$ tienen un enfoque de alto rendimiento.

b) Por titulaciones, el 57,65\% de los estudiantes de Pedagogía y el 59,57\% del alumnado de Informática manifiestan preferir un enfoque superficial. Por el contrario el 51,16\% de los estudiantes de Psicopedagogía y el 66,67\% del alumnado de Historia prefiere un enfoque de aprendizaje profundo.

c) Las variables de los estilos de aprendizaje preferidas por nuestra muestra son la extraversión (E), la percepción sensorial $(\mathrm{S})$, la toma de decisiones reflexiva $(\mathrm{T})$, y las funciones perceptivas $(\mathrm{P})$.

d) El mayor porcentaje de estudiantes con un enfoque superficial prefieren la extraversión (E), la percepción sensorial (S), la toma de decisiones reflexiva (T) y los procesos perceptivos (P).

e) El mayor porcentaje de estudiantes que tienen un enfoque de aprendizaje profundo prefiere la extraversión (E), la percepción sensorial $(\mathrm{S})$, tomar decisiones reflexivamente (T) y las funciones de juicio (J).

f) Son mayoría los estudiantes que tienen un enfoque de aprendizaje de alto rendimiento que prefieren la introversión (I), percibir preferentemente a través de los sentidos (S), tomar decisiones reflexivamente (T) y que se inclinan por las funciones de juicio (J).

g) Los motivos y las estrategias de los estudiantes con un enfoque superficial se relacionan con las funciones perceptivas y concretamente por la percepción sensorial (S).

h) Los motivos y las estrategias de los estudiantes con un enfoque profundo se relacionan con la intuición $(\mathrm{N})$, la reflexión (T), y la introversión (I).

i) Los motivos y estrategias de los estudiantes con un enfoque de alto rendimiento se relacionan con las funciones de juicio $(\mathrm{J})$.

j) Los estudiantes con enfoques de aprendizaje profundo y de alto rendimiento prefieren las funciones de juicio $(\mathrm{J})$. Es decir, utilizan menos tiempo en recabar información y más tiempo en tomar decisiones, solucionar problemas y obtener conclusiones.

k) Los estudiantes con enfoque de aprendizaje superficial se encuentran más cómodos utilizando las funciones perceptivas $(\mathrm{P})$. Emplean gran parte de su tiempo en recoger datos, información. Sus funciones preferentes no son las encargadas de tomar decisiones.

\section{Referencias bibliográficas}

Biggs, J. (1987). Student Approaches to learning and studying. Hawthorn, Vic.: Australian Council for Educational Research.

Biggs, J. (1999). Teaching for Quality Learning at University. SRHE y Open University.

Dunn, R., Dening, S., Lovelace, M. (2001). "Two sides of the same coin or different strokes for different folks?". Teacher Librarian, 28, 9-16. 
Gibbs, G. (1992). Improving the Quality of Student Learning. Bristol: Technical and Educational Service.

Hernández Pina, F. (1993). "Concepciones en el estudio del aprendizaje de los estudiantes universitarios”. Revista de Investigación Educativa, 22, pp. 117-150.

Hernández Pina, F. (1999). Los enfoques de aprendizaje en el contexto de la evaluación de la calidad de las universidades. Programa sectorial de promoción general del conocimiento. MEC. Convocatoria 1995. Informe final, 1999.

Hernández Pina, F., García, P., Martínez, P., Hervás, R. M., Maquilón, J. (2002). "Consistencia entre motivos y estrategias de aprendizaje en estudiantes universitarios". Revista de Investigación Educativa, 20, 2; 223-246.

Hervás Avilés, R. M. (2003). Estilos de enseñanza y de aprendizaje en escenarios educativos. Granada: GEU.

Hervás Avilés, R. M., Castejón Costa, J. (2003). “¿Están relacionados los modos de acceso al conocimiento y los tipos psicológicos? Análisis de su influencia en el logro académico". Bordón, 55 (4), 527-540.

Kember, D. y Gow, L. (1993). "Cultural specificity of approaches to study". Brithis Journal Eduactional Psychology, 60, pp. 228-234.

Kember, D., Wong, A. y Leung, D. Y. P. (1999). "Reconsidering the dimensions of approaches to learning”. British J. of Educational Psychology, 69, 323-343.

Kember, D. (2000). "Misconceptions about the learning approaches, motivation and study practices of Asian students". Higher Education, 40, 99-121.

Laurillard, D. (1984). "Learning from problems solving". En Marton, F., Hounsell, D. y Entwistle, N. (eds.). The Experience of Learning. Edimburgo: Scottish Academic Press

Marton, F. \& Säljö, R. (1976a). "On qualitative differences in learning I: Outcome and process". British Journal of Educational Psychology, 46, 4-11.

Marton, F. \& Säljö, R. (1976b). "On qualitative differences in learning II: Outcome as a function of the learner's conception of the task". British Journal of Educational Psychology, 46, 115-127.

Murray-Harvey, R. (1994). "Learning styles an approaches to learning: distinguishing between concepts and instruments". British Journal of Educational Psychology, 64, 373-388. Great Britain: The Brithis Psychological Society.

Myers, I. B., Briggs, K. (1943, 1976). Myers-Briggs type indicator. Palo Alto, Calif.: Consulting Psychologist Preess, Inc.

Myers, I. B. y McCaulley, M. H. (1985). Manual: A guide to the developement and use of the MyersBriggs Type Indicator. Palo Alto: Consulting Psychologist Press.

Ramsden, P. (1984). "The context of learning”. En Marton, F., Hounsell, D. y Entwistle, N. (eds.). The Experience of Learning. Edinburg: Scottish Academic Press.

Richardson, J. T. E. (1994). "Cultural specificity of approaches to studying in higher education a literature survey". Higher Education, 27, 449-468.

Sadler-Smith, E. (1997). "Learning style: frameworks and instruments". Educational Psychology, 17, 51-64.

Snyder, R. (2000). "The relationship between learning styles and multiple intelligences and academic achievement of high school students". High School Journal, 83, 11-21.

Trigwell, K., Prosser, M. (1996). "The intention to both memorise and understand: Another approach to learning?". High Education, 32, 341-354. 
Zhang, L. (2000). “Are thinking styles and personality types related?”. Educational Psychology, 20 (3), 271-284.

Zhang, L.(2001). "Approaches and thinking styles in teaching”. Journal of Psychology, 135, 547-561.

Zhang, L. (2003). "Does the five predict learning approaches?". Personality and individual differences, 34, 8, 1431-1446.

Zhang, L. (2004). "Do university studens' thinking styles matter in their preferred teaching approaches?". Personality and individual differences.

Zhang, L., \& Sternberg, R. (2000). "Are learning approaches and thinking styles related? A study in two chinese populations". Journal of Psychology Interdisciplinary and Applied, 134, 469-490.

Fecha de recepción: 20-06-04

Fecha de revisión: 04-06-05

Fecha de aceptación: 19-10-05 